\section{Insuficiencia renal en pacientes cardiológicos: buscar para encontrar}

\author{
DELICIA INÉS GENTILLE LORENTE ${ }^{1}$, TERESA SALVADÓ USACH ${ }^{\mathrm{a}}$
}

\section{Frequency of kidney failure in cardiologic patients: the need to search}

Background: Chronic kidney disease is a major health problem since it is associated with a high cardiovascular risk, total morbidity and mortality, increasing prevalence and high cost treatment. Aim: To assess the frequency of chronic kidney disease among patients consulting in a cardiology clinic. Material and Methods: Cross-sectional assessment of 649 patients attended at a cardiology clinic. Demographic, clinical, electrocardiographic, echocardiographic and laboratory variables were registered. Patients were considered to have a kidney failure when their estimated glomerular filtration rate was $<60 \mathrm{ml} / \mathrm{min} / 1.73$ $m^{2}$ according to the Modification of Diet in Renal Disease (MDRD) formula. Kidney failure was considered chronic if this alteration lasted $\geq 3$ months and hidden when serum creatinine levels were normal. Results: The frequency of kidney failure was $20.8 \%$ (28.4\% in patients $\geq 65$ years old). The mean age of patients with the disease was $71.5 \pm 9.1$ years and $52 \%$ were women. Eighty seven percent were in stage 3,10\% in stage 4 and $3 \%$ in stage 5. Among patients with kidney failure, in 114 (84\%) it was chronic and in 28\%, hidden. The latter was observed almost exclusively in women with creatinine levels of approximately 1 $m g / d l$. Hypertension (Odds ratio (OR) 4.2), age (OR 1.1), ventricular ejection fraction (OR 0.97) and low hemoglobin (OR 0.735) were the risk factors for kidney failure detected in the multivariate analysis. Conclusions: The frequency of kidney failure (chronic or hidden) was high in this group of cardiologic patients. Most patients had a mild to moderate failure and the risk factors were hypertension, age, low ventricular ejection fraction and low hemoglobin levels.

(Rev Med Chile 2015; 143: 1105-1113)

Key words: Atherosclerosis; Cardiovascular diseases; Renal insufficiency, chronic.
'Doctora en Medicina y Cirugía.

Especialista en Cardiología.

Servicio de Cardiología. Hospital de Tortosa Verge de la Cinta. IISPV. Tarragona, España. aDoctora en Biología. Diplomada en Estadística. Servicio de Anatomía Patológica. Hospital de Tortosa Verge de la Cinta. IISPV. Tarragona, España.

Recibido el 28 de octubre de 2014, aceptado el 6 de julio de 2015.

Correspondencia a: Delicia Inés Gentille Lorente C/ Esplanetes no 14. 43500 Tortosa (Tarragona). España. Teléfono: 0034977519105 dgentille.ebre.ics@gencat.cat
L a enfermedad renal crónica (ERC) constituye un importante problema de salud por su alta morbimortalidad, su creciente prevalencia dado el envejecimiento poblacional y el aumento de sus factores de riesgo, y los elevados costes que genera, resultando imprescindible su diagnóstico y tratamiento precoces.

La ERC se clasifica, según las guías de la Kidney Disease Outcomes Quality Initiative (KDOQI), en etapas de severidad según el filtrado glomerular (FG) y la existencia de lesión renal durante $\geq$ a 3 meses, definida como la presencia de anomalías es- tructurales o funcionales del riñón objetivadas directamente mediante el estudio histológico de una biopsia renal o, indirectamente, por la presencia de albuminuria, alteraciones en el sedimento urinario, alteraciones hidroelectrolíticas secundarias a patología tubular o a través de técnicas de imagen (Figura 1$)^{1,2}$. Se añaden tres categorías pronósticas según la albuminuria existente ${ }^{2}$. No obstante, es habitual denominar insuficiencia renal (IR) a la situación en la que el FG es $<60 \mathrm{ml} / \mathrm{min} / 1,73 \mathrm{~m}^{2}$ (estadios 3-5) e insuficiencia renal crónica (IRC) a la IR que persiste durante $\geq 3$ meses. Además, 


\begin{tabular}{|c|c|l|}
\hline $\begin{array}{c}\text { Estadio } \\
\text { ERC }\end{array}$ & $\begin{array}{c}\text { FG (ml/min/ } \\
\left.\mathbf{1 , 7 3} \mathbf{~ c m}^{\mathbf{2}}\right)\end{array}$ & \multicolumn{1}{|c|}{ DESCRIPCIÓN } \\
\hline 1 & $\geq 90$ & Daño renal con FG normal \\
\hline 2 & $60-89$ & $\begin{array}{l}\text { Daño renal y ligero descenso } \\
\text { del FG }\end{array}$ \\
\hline IRC \\
\hline $3 a$ & $45-59$ & $\begin{array}{l}\text { Descenso ligero-moderado } \\
\text { del FG }\end{array}$ \\
\hline $3 b$ & $30-44$ & Descenso moderado del FG \\
\hline 4 & $15-29$ & Descenso severo del FG \\
\hline 5 & $<15$ & Prediálisis o diálisis \\
\hline
\end{tabular}

Figura 1. Estadios de la clasificación K/DOQI de la ERC. * Los estadios 3-5 constituyen la, habitualmente, denominada insuficiencia renal crónica (IRC).

existe la llamada insuficiencia renal oculta (IRO) en la que, siendo los niveles séricos de creatinina normales, el FG es $<60 \mathrm{ml} / \mathrm{min} / 1,73 \mathrm{~m}^{2}$.

En España, los estudios de prevalencia de la IR realizados arrojan cifras significativas pero dispares. En población general, las tasas de afectación van de $13,1 \%{ }^{3,4}$ hasta $6,8 \%$ del estudio EPIRCE (el principal realizado en este ámbito, con 2.746 personas $\geq 20$ años $)^{5}$ resultando así similar a 4,7-8,1\% detectada en otros países europeos. En mayores de 64 años se han hallado prevalencias del 21,4 y $31 \%{ }^{5-7}$. Estudios en atención primaria reportan tasas de $7 \%$ (siendo $43,5 \%$ de los casos IRO $)^{8}$, de $14,5 \%{ }^{9}$, del $16,4 \%(26,1 \% \text { son IRO })^{10}$ y hasta del 21,3\% (estudio EROCAP con 7.202 pacientes, donde $37,3 \%$ fueron IRO $)^{11}$, encontrándose prevalencias de 21,2 y $33,7 \%$ en los mayores de 64 y 70 años respectivamente ${ }^{11}$. En cuanto a los pacientes hipertensos, se reportan prevalencias de $25,7 \%{ }^{12}$ y $30 \%$ con un tercio de los casos como IRO (estudio RICAR en 1.224 hipertensos) ${ }^{13,14}$. En las consultas de cardiología se han detectado tasas de 34\% (siendo IRO 48,2\%) ${ }^{15}$.

Múltiples estudios y metaanálisis han constatado que un FG reducido es un indicador de mayor riesgo de morbilidad y mortalidad cardiovascular (CV) y total. Los factores de riesgo CV y la enfermedad CV establecida llevan a la ERC y ésta, a su vez, lleva a la enfermedad $C V$, que constituye así su primera causa de muerte ${ }^{16-21}$. Es por esto que los pacientes con IRC son de alto o muy alto riesgo $\mathrm{CV}$ y que la IRC es considerada por muchos como un equivalente de la enfermedad coronaria, siendo así tributaria de medidas terapéuticas y objetivos de prevención secundaria $\mathrm{CV}^{15-17,21-24}, \mathrm{y}$ por eso, se recomienda su tamizaje especialmente en poblaciones con alto riesgo de padecerla ${ }^{22}$. La determinación del FG mediante fórmulas sencillas como la MDRD abreviada o, más recientemente, la CKD-EPI ${ }^{2}$ constituye el mejor método para estimar la función renal global.

\section{Objetivos}

Estudiar la prevalencia de la insuficiencia renal en pacientes cardiológicos.

\section{Metodología}

\section{Diseño del estudio}

Estudio descriptivo observacional transversal, con componente analítico y retrospectivo, que incluyó a pacientes de raza caucásica consecutivos $\geq 18$ años visitados en una consulta de cardiología del Hospital de Tortosa (Tarragona, España).

\section{Variables}

Las variables registradas se obtuvieron de la historia clínica así como de la anamnesis, exploración física y exámenes complementarios realizados a los pacientes. La analítica índice fue la más reciente realizada en situación basal dentro de los 12 meses previos a la consulta, y se consideró también la anterior a ésta realizada con diferencia de $\geq$ a 3 meses. El método de determinación de la creatinina fue el método Jaffé cinético, no estandarizado. Para estimar el FG se utilizó la fórmula MDRD-4 abreviada. Se consideró que existía una IR si el FG era $<60 \mathrm{ml} / \mathrm{min} / 1,73 \mathrm{~m}^{2}$, una IRC si la IR estaba presente en dos analíticas separadas por $\geq 3$ meses, y una IRO si la IR ocurría con niveles séricos de creatinina normales $(<1,2 \mathrm{mg} / \mathrm{dl}$ en mujeres, $<1,3 \mathrm{mg} / \mathrm{dl}$ en hombres).

\section{Análisis estadístico}

Se utilizó SPSS versión 16.0. Inicialmente, se realizó un estudio descriptivo expresando los resultados de las variables cuantitativas como media y desviación estándar $(\bar{x} \pm D E)$ y los de las variables cualitativas en porcentaje. Posteriormente, mediante regresión logística, se realizó análisis univariado y multivariado. Se calcularon los odds ratio (OR) ajustados y sus intervalos de confianza (IC) de 95\%. 
Para establecer determinadas relaciones entre dos variables se aplicaron los tests de t de Student, U de Mann-Whitney, Rho de Spearman y el estadístico exacto de Fisher. El nivel de significación (P) establecido fue de $<0,05$.

\section{Resultados}

Se incluyeron en el estudio a 649 pacientes. Sus características y antecedentes patológicos se resumen en la Tabla 1. El 71\% eran hipertensos, $65,8 \%$ dislipidémicos, $29,7 \%$ diabéticos y $16,3 \%$ fumaban. En cuanto a la enfermedad ateroescle- rótica, 31,7\% presentaban una cardiopatía isquémica, 35\% una vasculopatía periférica y 10,8\% una enfermedad vascular cerebral.

Se encontró que $135(20,8 \%)$ pacientes presentaban una IR, siendo su media de edad de 71,5 $\pm 9,1$ años (rango: 44 a 89 años) y $70(51,9 \%)$ de ellos mujeres. El 77,8\% de los casos tenían $\geq 65$ años (el grupo de 75 a 84 años registraba $44,4 \%$ de las IR) (Tabla 2). Los casos de IRC establecida fueron 114, suponiendo $84,4 \%$ de todas las IR.

Por estadios, $87,4 \%$ de las IR correspondían a un estadio 3 (3a el 63,7\%), 9,6\% a un estadio $4 \mathrm{y}$ $3 \%$ a 5 ; la media de edad de los afectados fue mayor en las mujeres, salvo en el estadio 5 (Tabla 3 ).

Tabla 1. Características y antecedentes de total de pacientes y del grupo con IR (FG $<60 \mathrm{ml} / \mathrm{min} / 1,73 \mathrm{~cm}^{2}$ ) y $\sin$ IR (FG $\geq 60 \mathrm{ml} / \mathrm{min} / 1,73 \mathrm{~cm}^{2}$ )

\begin{tabular}{|c|c|c|c|c|}
\hline & Total & Con IR & Sin IR & $\mathbf{p}$ \\
\hline \multicolumn{5}{|l|}{ Factores de riesgo $\mathrm{CV}$} \\
\hline \multicolumn{5}{|l|}{ Sexo $(\%)$} \\
\hline Hombres & $387(59,6)$ & $65(48,1)$ & $322(62,6)$ & \multirow[t]{2}{*}{$<0,0001$} \\
\hline Mujeres & $262(40,4)$ & $70(51,9)$ & $192(37,4)$ & \\
\hline Edad (años) $\bar{x} \pm D E$ & $64,6 \pm 13,1$ & $71,5 \pm 9,1$ & $63,4 \pm 13,0$ & $<0,0001$ \\
\hline HTA (\%) & $461(71,0)$ & $115(85,2)$ & $344(66,9)$ & $<0,0001$ \\
\hline Dislipemia (\%) & $427(65,8)$ & $104(77,0)$ & $323(62,8)$ & $<0,0001$ \\
\hline Diabetes Mellitus (\%) & $193(29,7)$ & $55(40,7)$ & $138(26,8)$ & 0,003 \\
\hline $\mathrm{IMC}\left(\mathrm{Kg} / \mathrm{m}^{2}\right) \overline{\mathrm{x}} \pm \mathrm{DE}$ & $28,7 \pm 4,8$ & $28,9 \pm 4,4$ & $28,7 \pm 5,0$ & 0,722 \\
\hline \multicolumn{5}{|l|}{ Tabaco (\%) } \\
\hline No fumador & $371(57,1)$ & $86(63,7)$ & $285(55,4)$ & \multirow[t]{3}{*}{0,041} \\
\hline Fumador & $106(16,3)$ & $13(9,6)$ & $93(18,1)$ & \\
\hline Exfumador & $172(26,4)$ & $36(26,7)$ & $136(26,5)$ & \\
\hline \multicolumn{5}{|l|}{ ECG: ritmo cardiaco (\%) } \\
\hline Sinusal & $532(82,0)$ & $102(75,5)$ & $430(83,6)$ & \multirow[t]{3}{*}{ n.a. } \\
\hline Fibrilación auricular & $112(17,2)$ & $33(24,4)$ & $79(15,4)$ & \\
\hline Otros & $5(0,8)$ & $0 \quad(0,0)$ & $5(1,0)$ & \\
\hline \multicolumn{5}{|l|}{ Enfermedad CV } \\
\hline \multicolumn{5}{|l|}{ Cardiopatía isquémica (\%) } \\
\hline No & $443(68,3)$ & $77(57,0)$ & $366(71,2)$ & 0,003 \\
\hline Asintomática & $14(2,2)$ & $5(3,7)$ & $9(1,7)$ & 0,187 \\
\hline Angor & $76(11,7)$ & $20(14,8)$ & $56(10,9)$ & 0,233 \\
\hline IAM & $116(17,9)$ & $33(24,4)$ & $83(16,1)$ & 0,026 \\
\hline \multicolumn{5}{|c|}{ Enfermedad cerebrovascular (\%) } \\
\hline No & $579(89,2)$ & $114(84,4)$ & $465(90,5)$ & 0,053 \\
\hline AIT & $17(2,6)$ & $6(4,4)$ & $11(2,1)$ & 0,142 \\
\hline Ictus & $53(8,2)$ & $15(11,1)$ & $38(7,4)$ & 0,178 \\
\hline \multicolumn{5}{|c|}{ Enfermedad vascular periférica (\%) } \\
\hline No & $422(65,0)$ & $62(45,9)$ & $360(70,0)$ & $<0,0001$ \\
\hline Soplo femoral & $89(13,7)$ & $25(18,5)$ & $64(12,5)$ & 0,056 \\
\hline Claudicación intermitente & $23(3,5)$ & $9(6,7)$ & $14(2,7)$ & 0,030 \\
\hline
\end{tabular}


Tabla 1. Continuación

\begin{tabular}{|c|c|c|c|c|}
\hline & Total & Con IR & Sin IR & $\mathbf{p}$ \\
\hline \multicolumn{5}{|c|}{ Estudio ecocardiográfico } \\
\hline HVI (\%) & $68(10,5)$ & $16(11,9)$ & $52(10,1)$ & 0,575 \\
\hline $\mathrm{FEVI} \overline{\mathrm{x}} \pm \mathrm{DE}$ & $58,9 \pm 11,9$ & $56,4 \pm 13,2$ & $59,2 \pm 11,6$ & 0,050 \\
\hline$E / e^{\prime} \bar{x} \pm D E$ & $11,3 \pm 6,9$ & $13,3 \pm 10,3$ & $10,7 \pm 5,2$ & 0,003 \\
\hline \multicolumn{5}{|c|}{ Disfunción diastólica (\%) } \\
\hline Grados 0 y I & $473(85,4)$ & $95(83,3)$ & $378(85,9)$ & 0,488 \\
\hline Grados II, III y IV & $81(14,6)$ & $19(16,7)$ & $62(14,1)$ & \\
\hline \multicolumn{5}{|c|}{ Dilatación de la Al (\%) } \\
\hline No & $353(55,8)$ & $59(44,0)$ & $294(58,9)$ & 0,002 \\
\hline Sí & $280(44,2)$ & $75(56,0)$ & $205(41,1)$ & \\
\hline \multicolumn{5}{|c|}{ Esclerosis válvula aórtica (\%) } \\
\hline No y no severa & $596(98,3)$ & $120(96,8)$ & $476(98,8)$ & 0,127 \\
\hline Severa & $10(1,7)$ & $4(3,2)$ & $6(1,2)$ & \\
\hline \multicolumn{5}{|c|}{ Calcificación anillo mitral (\%) } \\
\hline No y No severa & $619(98,3)$ & $128(97,7)$ & $490(98,4)$ & 0,706 \\
\hline Severa & $11 \quad(1,7)$ & $3(2,3)$ & $8(1,6)$ & \\
\hline \multicolumn{5}{|c|}{$\begin{array}{l}\text { Dimensiones de la aorta }(\mathrm{mm}) \\
\overline{\mathbf{x}} \pm \mathrm{DE} \text { (rango) }\end{array}$} \\
\hline Raíz & $34,0 \pm 4,7(22-48)$ & $33,4 \pm 4,8(25-48)$ & $34,2 \pm 4,6(22-48)$ & 0,053 \\
\hline Aorta ascendente & $33,9 \pm 5,2(20-50)$ & $34,3 \pm 4,9(20-47)$ & $34,0 \pm 5,3(20-50)$ & 0,527 \\
\hline Aorta infrarrenal & $17,9 \pm 4,1(9-39)$ & $19,1 \pm 8,5(9-37)$ & $18,2 \pm 4,7(10-39)$ & 0,916 \\
\hline \multicolumn{5}{|c|}{ Análisis sanguíneo $\bar{x} \pm \mathrm{DE}$} \\
\hline Glucosa (mg/dl) & $110,1 \pm 33,5$ & $116,4 \pm 41,0$ & $108,5 \pm 31,2$ & 0,157 \\
\hline Hemoglobina ( $g / d l)$ & $13,8 \pm 1,8$ & $13,1 \pm 1,9$ & $14,1 \pm 1,7$ & $<0,0001$ \\
\hline $\mathrm{Hb}$ A1C (\%) & $6,0 \pm 1,8$ & $6,6 \pm 2,1$ & $5,8 \pm 1,7$ & 0,010 \\
\hline Creatinina (mg/dl) & $1,0 \pm 0,7$ & $1,6 \pm 1,2$ & $0,9 \pm 0,2$ & $<0,0001$ \\
\hline$c-L D L(m g / d l)$ & $119,2 \pm 35,7$ & $114,6 \pm 37,9$ & $120,4 \pm 35,0$ & 0,129 \\
\hline $\mathrm{c}-\mathrm{HDL}(\mathrm{mg} / \mathrm{dl})$ & $42,8 \pm 16,4$ & $42,2 \pm 15,6$ & $42,6 \pm 15,2$ & 0,834 \\
\hline $\mathrm{TG}(\mathrm{mg} / \mathrm{dl})$ & $119,6 \pm 79,5$ & $123,0 \pm 96,2$ & $116,7 \pm 73,7$ & 0,090 \\
\hline
\end{tabular}

CV: cardiovascular; c-LDL: colesterol LDL; c-HDL: colesterol HDL; ECG: electrocardiograma; FEVI: fracción de eyección del VI; HVI: hipertrofia ventricular izquierda; HTA: hipertensión arterial; IAM: infarto agudo de miocardio; IMC: índice de masa corporal; TG: triglicéridos.

Tabla 2. Prevalencia de la IR por grupos de edad

\begin{tabular}{|cccc|}
\hline $\begin{array}{c}\text { Grupos de edad } \\
\text { (años) }\end{array}$ & $\begin{array}{c}\text { Con IR } \\
\mathbf{n}(\%)\end{array}$ & $\begin{array}{c}\text { Sin IR } \\
\mathbf{n}(\%)\end{array}$ & Total \\
\hline 65 & $30(10,7)$ & $250(89,3)$ & 280 \\
\hline $65-74$ & $42(21,4)$ & $154(78,6)$ & 196 \\
\hline $75-84$ & $60(35,9)$ & $107(64,1)$ & 167 \\
$\geq 85$ & $3(50,0)$ & $3(50,0)$ & 6 \\
\hline Total & $135(20,8)$ & $514(79,2)$ & 649 \\
\hline
\end{tabular}

IR: insuficiencia renal. 
Tabla 3. Prevalencia de la IR según estadios KDOQI y sexo

\begin{tabular}{|c|c|c|c|}
\hline $\begin{array}{c}\text { Estadio de IRC } \\
\left(\mathrm{ml} / \mathrm{min} / 1,73 \mathrm{~cm}^{2}\right)\end{array}$ & $\begin{array}{c}\text { Hombres (\%) } \\
\text { Edad (años) } \bar{x} \pm \text { DE }\end{array}$ & $\begin{array}{c}\text { Mujeres (\%) } \\
\text { Edad (años) } \bar{x} \pm \text { DE }\end{array}$ & $\begin{array}{c}\text { Total (\%) } \\
\text { Edad (años) } \bar{x} \pm \text { DE }\end{array}$ \\
\hline 3. FG 30-59 & $\begin{array}{c}51(78,5) \\
68,9 \pm 9,0\end{array}$ & $\begin{array}{c}67(95,7) \\
74,7 \pm 8,2\end{array}$ & $\begin{array}{l}118(87,4) \\
72,2 \pm 9,0\end{array}$ \\
\hline 3а. FG 45-59 & $\begin{array}{c}35(53,8) \\
68,5 \pm 7,6\end{array}$ & $\begin{array}{c}51(72,8) \\
73,0 \pm 8,6\end{array}$ & $\begin{array}{c}86(63,7) \\
71,1 \pm 8,5\end{array}$ \\
\hline 3b. FG 30-44 & $\begin{array}{c}16(24,6) \\
69,3 \pm 11,7\end{array}$ & $\begin{array}{c}16(22,8) \\
77,6 \pm 7,0\end{array}$ & $\begin{array}{c}32(23,7) \\
73,6 \pm 10,2\end{array}$ \\
\hline 4. FG 15-29 & $\begin{array}{c}11(16,9) \\
68,1 \pm 9,4\end{array}$ & $\begin{array}{c}2(2,85) \\
75,0 \pm 0,0\end{array}$ & $\begin{array}{c}13(9,6) \\
69,2 \pm 9,0\end{array}$ \\
\hline 5. FG $<15$ o diálisis & $\begin{array}{c}3(4,6) \\
85,0 \pm 11,4\end{array}$ & $\begin{array}{c}1(1,4) \\
66,0\end{array}$ & $\begin{array}{c}4(3,0) \\
66,8 \pm 10,1\end{array}$ \\
\hline Total & 65 & 70 & $\begin{array}{c}135 \\
71,5 \pm 9,1\end{array}$ \\
\hline
\end{tabular}

FG: filtrado glomerular; IRC: insuficiencia renal crónica.

Tabla 4. Número de pacientes con los distintos niveles de colesterol LDL según el estadio de IR

\begin{tabular}{|c|c|c|c|c|}
\hline & $\begin{array}{c}\text { Estadio } 3 \\
(\%)\end{array}$ & $\begin{array}{c}\text { Estadio } 4 \\
(\%)\end{array}$ & $\begin{array}{c}\text { Estadio } 5 \\
(\%)\end{array}$ & $\begin{array}{l}\text { Total } \\
\text { n (\%) }\end{array}$ \\
\hline $\mathrm{cLDL}<100$ & $34(82,9)$ & $4 \quad(9,8)$ & $3(7,3)$ & $41 \quad(36,9)$ \\
\hline $\mathrm{cLDL} \geq 100$ & $63(90,0)$ & $7(10,0)$ & $0 \quad(0,0)$ & $70 \quad(63,1)$ \\
\hline Total & $97(87,4)$ & $11 \quad(9,9)$ & $3(2,7)$ & $111(100,0)$ \\
\hline $\mathrm{cLDL}<70$ & $6(66,7)$ & $1(11,1)$ & $2(22,2)$ & $9 \quad(8,1)$ \\
\hline $\mathrm{cLDL} \geq 70$ & $91(89,2)$ & $10 \quad(9,8)$ & $1(1,0)$ & $102(91,9)$ \\
\hline Total & $97(87,4)$ & $11 \quad(9,9)$ & $3(2,7)$ & $111(100,0)$ \\
\hline
\end{tabular}

c-LDL: colesterol LDL.

La IR se asoció significativamente al género, la edad, la HTA, la diabetes, la dislipidemia, el hábito tabáquico, la cardiopatía isquémica, la vasculopatía periférica, la dilatación auricular izquierda, E/e' (parámetro ecocardiográfico que valora la función diastólica del ventrículo izquierdo; normal es $<8$ ) y los niveles de hemoglobina. Respecto a la HTA, la prevalencia de IR en los hipertensos fue de $24,9 \%$ mientras que $85,2 \%$ de los IR eran hipertensos. En cuanto a la dislipidemia, $36,9 \%$ de los pacientes con IR presentaban c-LDL $<100 \mathrm{mg} / \mathrm{dl}$ y $8,1 \%$ niveles $<70 \mathrm{mg} / \mathrm{dl}$ (Tabla 4 ).

Los resultados del análisis univariado, realizado con todas las variables, y del multivariado, realizado con aquellas con $\mathrm{p}<0,10$ en el univa- riado, se muestran en Tablas 5 y 6 .

De todas la IR, $38(28,1 \%)$ fueron una IRO: 37 $(97,3 \%)$ eran mujeres, con media de edad de 74,8 $\pm 9,4$ años, y todas correspondían a un estadio 3; así, en las mujeres, 55,2\% de las IR estadio 3 eran una IRO, no existiendo diferencias significativas entre ambos grupos en cuanto a cada una de las variables de la Tabla 2 . El valor de creatinina para el que el FG era ya $<60 \mathrm{ml} / \mathrm{min}$ fue de 1 $\mathrm{mg} / \mathrm{dl}$. Al considerar como normales los valores de creatinina $<1 \mathrm{mg} / \mathrm{dl}$, la sensibilidad y el valor predictivo negativo para detectar una IR fueron de $100 \%$ (frente a $46,4 \%$ y $83,8 \%$ respectivamente de la serie para un límite corte de creatinina de $<1,2 \mathrm{mg} / \mathrm{dl}$ ). 
Tabla 5. Análisis univariante. Odds ratio de las variables significativas en la IR

\begin{tabular}{|lcccc|}
\hline Variable & Referencia & p & Odds ratio & IC 95\% \\
\hline Sexo & Hombre & 0,003 & 1,779 & $1,215-2,603$ \\
\hline Edad & - & $<0,0001$ & 1,072 & $1,049-1,095$ \\
\hline HTA & No & $<0,0001$ & 2,857 & $1,718-4,752$ \\
\hline Dislipemia & No & 0,005 & 1,844 & $1,199-2,837$ \\
\hline Diabetes & No & 0,003 & 1,813 & $1,223-2,687$ \\
\hline Fumador & No & 0,065 & - & - \\
\hline Cardiopatía isquémica & No & 0,003 & 1,802 & $1,219-2,665$ \\
\hline IAM & No & 0,027 & 1,676 & $1,061-2,648$ \\
\hline Enfermedad vascular periférica & No & $<0,0001$ & 2,125 & $1,410-3,202$ \\
\hline Claudicación intermitente & No & 0,030 & 2,511 & $1,063-5,932$ \\
\hline Ausencia de pulsos distales o ITB anómalo & No & $<0,0001$ & 2,404 & $1,538-3,759$ \\
\hline FEVI & - & 0,018 & 0,981 & $0,966-0-997$ \\
\hline E/e' & - & 0,011 & 1,054 & $1,012-1,098$ \\
\hline Dilatación de la Al & No & 0,002 & 1,823 & $1,241-2,679$ \\
\hline Hemoglobina & - & $<0,0001$ & 0,734 & $0,657-0,821$ \\
\hline HbA1C & - & 0,015 & 1,242 & $1,043-1,480$ \\
\hline Creatinina & - & $<0,0001$ & $5.882,154$ & $1.221,2-28.333,1$ \\
\hline
\end{tabular}

FEVI: fracción de eyección del VI; HTA: hipertensión arterial; IAM: infarto agudo de miocardio.

Tabla 6. Análisis multivariante. Odds ratio de las variables significativas en la IR ajustadas por las otras variables

\begin{tabular}{|lcccc|}
\hline Variable & Referencia & p & Odds ratio & IC 95\% \\
HTA & No & 0,004 & 4,150 & $1,582-10,887$ \\
Edad & - & 0,001 & 1,060 & $1,023-1,098$ \\
FEVI & - & 0,027 & 0,972 & $0,948-0,997$ \\
Hemoglobina & - & 0,001 & 0,735 & $0,614-0,880$ \\
\hline
\end{tabular}

FEVI: fracción de eyección del VI; HTA: hipertensión arterial.

\section{Discusión}

En una consulta de cardiología, la prevalencia de la IR fue de 20,8\% (28,4\% en los de $\geq$ a 65 años), siendo la media de edad de los afectados de 71,5 \pm 9,1 años, $51,9 \%$ mujeres y $87,4 \%$ una IR estadio 3 . De todas las IR, $84,4 \%$ eran ya una IRC establecida y $28,1 \%$ una IRO.

Al comparar estos resultados con los de trabajos previos, cabe matizar que los estudios de prevalencia de la IRC realizados hasta la fecha han sido transversales y, faltando al criterio temporal que la define, han basado su estimación en un único análisis sanguíneo (y no en dos y separados por $\geq 3$ meses) de modo que, en realidad, se trata de prevalencias de IR sin diferenciar entre una afectación renal transitoria y una IRC establecida. Adicionalmente, no existe uniformidad metodológica entre los distintos estudios y así, por ejemplo, se consideran distintos niveles de normalidad de creatinina cuya determinación, además, no está estandarizada y se usan diferentes fórmulas para 
estimar el FG. Teniendo en cuenta dichas circunstancias, se observa como la prevalencia de la IR en la serie es de $20,8 \%$ (inferior a la reportada) ${ }^{16}$ y la de la IRC de $17,6 \%$, mientras que en $\geq$ a los 65 años es de 28,4\% (similar a la detectada en éstos en población general ${ }^{5-7} y$ atención primaria $)^{11}$.

En cuanto a la severidad de la IR, y tal como reportan otros autores ${ }^{15}$, la mayoría de los casos (87,4\% de las IR) corresponden en ambos géneros a un estadio 3, principalmente a un 3a (IR leve). De este modo, el diagnóstico de la enfermedad suele ser en fases tempranas que es cuando las intervenciones terapéuticas para enlentecer su progresión y reducir sus complicaciones $\mathrm{CV}$ y totales son más efectivas ${ }^{22}$.

La asociación de la ERC, especialmente de los estadios 3-5, con la afectación CV ha sido demostrada en numerosos estudios realizados en población general, en población asistida en atención primaria, nefrópatas, hipertensos, pacientes con insuficiencia cardiaca o cardiopatía isquémica $y$ en pacientes hospitalizados, entre otros ${ }^{12}$. Sin embargo, en España no se disponen de estudios de prevalencia de la IR, IRC y morbimortalidad $\mathrm{CV}$ en los distintos pacientes que son atendidos diariamente en consultas de cardiología. Igualmente, y aunque en los últimos años se ha ido tomando consciencia de la transcendencia de la ERC en el pronóstico CV, el seguimiento de las recomendaciones actuales en cuanto al manejo de estos pacientes dista de ser el deseable en numerosos ámbitos asistenciales, incluida la cardiología ${ }^{24}$.

La mayor morbimortalidad CV de los pacientes con ERC se debe a una mayor prevalencia de los factores de riesgo CV tradicionales (mayor cuanto menor es el FG) y de factores de riesgo relacionados con su nefropatía (alteraciones del metabolismo mineral, calcificación arterial, disfunción endotelial, inflamación, anemia, etc. $)^{11,22}$, añadiéndose el hecho de que, en ocasiones, la presencia de una IR conlleva un manejo distinto y a un subtratamiento (menor uso de IECAs, beta bloqueantes, antitrombóticos e intervencionismo coronario, etc. $)^{16}$. Así, en la serie, se constata la existencia de una asociación significativa entre la IR y factores de riesgo CV como la edad, la HTA (presente en 85,2\% de las IR), la dislipidemia (77\% eran dislipidémicos) y la diabetes mellitus (presente en $40,7 \%$ ), y con enfermedades ateroescleróticas como la cardiopatía isquémica (relación con el infarto de miocardio) y la enfermedad vascular periférica (relación con la claudicación intermitente y pulsos distales anómalos). La población más estudiada tanto en atención primaria como en cardiología es la de los hipertensos, reportándose tasas de IR de $25,7 \%{ }^{12}$ a $30 \%{ }^{14}$ respectivamente; igualmente, en esta serie se observa como la IR afecta a $25,4 \%$ de los hipertensos mientras que la HTA afecta hasta a 85,2\% de los insuficientes renales. Estos datos ponen de manifiesto cómo la HTA constituye un factor de riesgo fundamental de la IR, como así confirma el análisis multivariado. Cabe resaltar que, en relación con el hábito tabáquico, los pacientes con IR sean menos fumadores activos que aquellos con FG $>60 \mathrm{ml} / \mathrm{min}$, hecho ya observado en el estudio EPIRCE y EROCAP ${ }^{5-11}$. De hecho, los únicos factores de riesgo independientes de la IR detectados son la HTA (OR 4,150), la edad (OR 1,060), el descenso de la FEVI (OR 0,972) y el descenso de los niveles de hemoglobina en sangre (OR 0,735) (Tabla 5). Estos resultados coinciden con estudios previos realizados en población general, en población de atención primaria o en hipertensos ${ }^{5,11,13}$ en los que la edad y la HTA son factores de riesgo independientes, mientras que en el EROCAP ${ }^{11}$ lo son la edad, la reducción de la hemoglobina y el antecedente de insuficiencia cardiaca (esto último sería un equivalente a la disminución de la FEVI de la serie). De hecho, se describe que $50 \%$ de pacientes con IC presentan algún grado de IR y que $40 \%$ de pacientes con IR presentan una IC, siendo la relación entre ambas bidireccional, multifactorial y, a menudo, con el componente añadido de una anemia ${ }^{25}$.

En cuanto a la ecocardiografía, resulta llamativo que a pesar de la estrechísima relación existente entre la IR, la HTA y la edad, los pacientes con IR no presentan más HVI, disfunción diastólica ni dilatación aórtica que aquellos sin IR, dado que la prevalencia descrita de HVI ecocardiográfica en hipertensos es muy variable (20 a 49\%). Los resultados de la serie podrían explicarse, al menos en parte, por ser la mayoría de las IR ligeras-moderadas (y secundariamente tener menor afectación de órgano diana), porque éstos pacientes pudieran tener un mejor control de sus cifras tensionales, o por lo criterios de HVI considerados (grosor de pared ventricular $>11 \mathrm{~mm}$ ).

En base a las recomendaciones actuales, los pacientes con IRC deben ser considerados como pacientes de muy alto riesgo CV. Por ello, y para reducir los eventos $\mathrm{CV}$ y ralentizar la pérdida de 
función renal, se impone el conseguir objetivos de prevención secundaria mediante cambios en el estilo de vida, la dieta y el tratamiento farmacológico. En cuanto a la dislipidemia, se recomienda prescribir estatinas (preferiblemente de eliminación hepática) y lograr niveles de c-LDL $<70 \mathrm{mg} /$ $\mathrm{dl}$, medida efectiva en estadios 2-5, excepto en el caso de pacientes ya en diálisis ${ }^{21,22,26,27}$. Sin embargo, en la serie se observa que, en los pacientes con IR y a pesar de tener $84,4 \%$ de ellos ya una IRC, apenas $36,9 \%$ tienen un c-LDL $<100 \mathrm{mg} / \mathrm{d}$ y tan sólo $8,1 \%$ de $<70 \mathrm{mg} / \mathrm{dl}$, objetivos que distan de ser los deseables.

Finalmente, con respecto a la IRO, es sabido que el estimar la función renal en base a la creatinina plasmática conlleva una alta tasa de IRO puesto que sus niveles varían por diversos factores (edad, género, peso o masa muscular, dieta, etc.) existiendo una relación hiperbólica abierta entre ambas variables ${ }^{28}$. Estudios en diversos ámbitos asistenciales muestran como la IRO constituye el 26 a 49,3\% de todas las IR, siendo mayoritariamente estadios 3 y afectando casi exclusivamente a ancianos y mujeres ${ }^{6-11,13,29}$. Los resultados de la serie son coincidentes con los previos: casi un tercio de las IR son una IRO, todas un estadio 3, y casi todas son mujeres y más ancianas que el resto de pacientes con IR. Estos resultados llevan a enfatizar en la necesidad de calcular el FG en la práctica clínica mediante fórmulas simples, especialmente en las mujeres y ancianos (población ésta muy a menudo polimedicada y con fármacos que requieren ajuste de dosis o que están contraindicados en la IR), y a plantear el descender el umbral de normalidad de creatinina a $<1 \mathrm{mg} / \mathrm{dl}$ en la mujer, lo que ofrece una sensibilidad y valor predictivo negativo de $100 \%$ para detectar una IR.

\section{Conclusiones}

La prevalencia de la IR, y de la IRC establecida, en pacientes cardiológicos es elevada y casi un tercio de los casos son una IRO. La mayoría de los casos corresponden a IR ligeras-moderadas. Los factores de riesgo independientes de la IR son la presencia de HTA, la edad, la menor FEVI y los menores niveles de hemoglobina. La detección de una IR permite, además, mejorar la estratificación CV de los pacientes pudiendo así tener importantes implicancias diagnósticas, pronósticas y terapéuticas.

\section{Referencias}

1. K/DOQI Clinical practice guidelines for chronic kidney disease evaluation, classification and stratification. Am J Kidney Dis 2002; 39 (Suppl 1): S1-S266.

2. KDIGO 2012 Clinical practice guideline for the evaluation and management of chronic kidney disease. Kidney int (Suppl) 2013; 3: 9-62.

3. Abelleira A, Otero A, Gayoso P. Enfermedad renal crónica oculta y factores de riesgo vascular asociados. Estudio epidemiológico. Nefrología 2005; 25: 275-87.

4. Simal F, Martín JC, Bellido J, Arzúa D, Mena FJ, González I, et al. Prevalencia de la enfermedad renal crónica leve y moderada en población general. Estudio Hortega. Nefrología 2004; 24: 329-37.

5. Otero A, Francisco A, Gayoso P, García F. Prevalencia de la insuficiencia renal crónica en España: Resultados del estudio EPIRCE. Nefrología 2010; 30: 78-86.

6. Almirall J, Vaqueiro M, Antón E, Baré ML, González V, Jaimez E, et al. Prevalencia de la insuficiencia renal en la población general mayor de 64 años y episodios cardiovasculares asociados. Nefrología 2005; 25: 655-62.

7. Cea-Calvo L, Redon J, Marti-Canales JC, Lozano JV, Llisterri JL, Fernández-Pérez C. Prevalencia de filtrado glomerular disminuido en la población española de edad avanzada. Med Clin (Barc) 2007; 129: 681-7.

8. Labrador PJ, Mengotti T, Jiménez M, Macías M, Vicente F, Labrador J, et al. Insuficiencia renal oculta en atención primaria. ¿Un problema exclusivo de mujeres? Nefrología 2007; 27 (6): 716-20.

9. Rodrigo MP, Andrés MR. Detección de insuficiencia renal oculta en consulta de atención primaria mediante la aplicación de la ecuación MDRD-abreviada: análisis de 1.000 pacientes. Nefrología 2006; 26: 339-43.

10. Lou LM, Campos B, Boned B, Turón JMa, Gimeno JA. Estimación del filtrado glomerular en atención primaria: prevalencia de enfermedad renal crónica y repercusión sobre la derivación a nefrología. Nefrología 2008; 28 : 329-32.

11. De Francisco ALM, De la Cruz JJ, Cases A, De la Figuera M, Egocheaga MI, Gorriz JI. Prevalencia de insuficiencia renal en Centros de Atención Primaria en España: Estudio EROCAP. Nefrología 2007; 27: 300-12.

12. Vara-González L, Martín E, Ureña T, Dalfó, Flor I, López Fernández V. Prevalencia de la enfermedad renal crónica en hipertensos seguidos en los centros de salud de España y grado de control de su presión arterial (estudio DISEHTAE). Atención Primaria 2008; 40: 241-5.

13. Fácila L, Bertomeu-González V, Bertomeu-Martínez V, González-Juanatey JR, Mazón P, Morillas P, en re- 
presentación de los investigadores del estudio RICAR. Importancia de la detección de la enfermedad renal oculta en pacientes hipertensos. Rev Esp Cardiol 2009; 62: 282-7.

14. Bertomeu-Martínez M, González-Juanatey JR, Bertomeu-González V, Fácila L, Mazón P, Quiles J, et al. Infravaloración del riesgo renal en consultas de cardiología. Estudio RICAR. Nefrología 2008; 28: 621-6.

15. Cases A, González-Juanatey JR, Conthe P, Matalí A, Garrido C. Prevalencia de insuficiencia renal crónica en pacientes de alto riesgo o con enfermedad cardiovascular. Rev Esp Cardiol 2010; 63: 225-8.

16. Schiffrin EL, Lipman ML, Mann JF. Chronic kidney disease: effects on the cardiovascular system. Circulation 2007; 116: 85-97.

17. Bardají A, Martínez-Vea A. Enfermedad renal crónica y corazón. Un continuo evolutivo. Rev Esp Cardiol 2008; 61: 41-51.

18. Go AS, Chertow GM, Fan D, McCulloch CE, Hsu C. Chronic kidney disease and the risks of death, cardiovascular events and hospitalization. N Engl J Med 2004; 351: 1296-305.

19. Weiner DE, Tighiouart H, Amin MG, Stark PC, MacLeod B, Griffith JL, et al. Chronic kidney disease as a risk factor for cardiovascular disease and all-cause mortality: a pooled analysis of community-based studies. J Am Soc Nephrol 2004; 15: 1307-15.

20. Weiner DE, Tighiouart H, Stark PC, Amin MG, MacLeod B, Griffith JL, et al. Kidney disease as a risk factor to recurrent cardiovascular disease and mortality. Am J Kidney Dis 2004; 44: 198-206.

21. Perk J, De Backer G, Gohlkea H, Graham I, Reiner Z, Verschuren M, et al. European guidelines on cardiovas- cular disease prevention in clinical practice. Eur Heart J 2012; 33: 1635-701.

22. Documento de consenso sobre la enfermedad renal crónica. SEN, SEMI, SEC, SEEN, SEA SHE, SEMFYC, SEMG, SEMERGEN, SEQC. 2012. www.senefro.org/ modules.php?name=news\&d_op=detail\&idnew $=1274$

23. Gorostidi. La insuficiencia renal como nuevo factor de riesgo cardiovascular. Riesgo vascular ligado a al microalbuminuria. Nefrología 2004; 24 (Supl 6): 47-61.

24. Gorostidi M, Santamaría R, Alcázar R, Fernández-Fresnedo G, Galcerán JM, Goicoechea M, et al. Documento de la Sociedad Española de Nefrología sobre las guías KDIGO para la evaluación y el tratamiento de la enfermedad renal crónica. Nefrología 2014; 34 (3): 302-16.

25. Martín A, Cordero A, Rodríguez M. Importancia del estudio de la función renal en cardiología. Med Clin (Barc) 2007; 128: 705-10.

26. Martín AL, Aguilera L, Fuster V. Enfermedad cardiovascular, enfermedad renal y otras enfermedades crónicas. Es necesaria una intervención más temprana en la enfermedad renal crónica. Nefrología 2009; 29: 6-9.

27. KDIGO Clinical practice guideline for lipid management in chronic kidney disease. Kidney int (Suppl) 2013; 3: 259-305.

28. Fernández Fresnedo G, de Francisco ALM, Rodrigo E, Herry I, Ruiz C. Insuficiencia renal "oculta” por valoración de la función renal mediante creatinina sérica. Nefrol 2002; 22: 144-51.

29. Rodríguez-Poncelas A, Quesada M, Coll G, Caula J, Gelada-Batlle, Gómez-Marcos MA, et al. Prevalencia de insuficiencia renal oculta y variables asociadas en una población de pacientes con diabetes tipo 2. Med Clin (Barc) 2010; 134: 239-45. 